\title{
CONTINUITY OF MULTIDIMENSIONAL BROWNIAN LOCAL TIMES
}

\author{
SHEY SHIUNG SHEU
}

(Communicated by George C. Papanicolaou)

\begin{abstract}
The local time of a multidimensional semimartingle at a hypersurface will be defined via Tanaka's formula. One can define a certain distance between hypersurfaces so that the continuity properties of local time can be discussed when the underlying process is Brownian motion.
\end{abstract}

\section{INTRODUCTION}

The local time of one-dimensional Brownian motion is very important additive functional in the construction of a general diffusion; see Itô and McKean [6] or Freedman [4]. That such construction is possible depends, besides others, on the fact that the local time can be made jointly continuous in the state and the time variables. As for the multidimensional case the local time at a state does not exist because Brownian motion never hits points. Nevertheless one can define in a natural way the local time at certain $(d-1)$-dimensional sets when the state space has dimension $d$. By introducing a metric between these sets, the continuity properties can be discussed when the underlying process is Brownian motion. Bass [1] has dealt with similar problems in a more general set up. Our approach here is more direct and specific. The main tool is Tanaka's formula. We shall employ this formula to handle continuity in an explicit manner. For other related works, see Yor [12].

\section{DEFINITION AND MOMENT INEQUALITIES}

Recall that if $X=\left(X_{t}\right)$ is a real continuous semimartingale, the local time of $X$ at a point $a$, denoted by $L_{t}^{a}(X)$, is defined through the Tanaka formula

$$
\left(X_{t}-a\right)^{+}=\left(X_{0}-a\right)^{+}+\int_{0}^{t} I_{\left[X_{s}>a\right]} d X_{s}+\frac{1}{2} L_{t}^{a}(X),
$$

$w$ here $x^{+}=w \vee 0, I_{A}$ is the indicator function of the set $A$; see Meyer [9]. If $X=\left(X^{1}, X^{2}, \ldots, X^{d}\right)$ is an $d$-dimensional continuous semimartingale (each coordinate is a real continuous semimartingale) and if $C$ is a class $C^{2}$ hypersurface; i.e., $C=\left\{x=\left(x_{1}, x_{2}, \ldots, x_{d}\right): x_{d}=g\left(x_{1}, x_{2}, \ldots, x_{d-1}\right)\right\}$,

Received by the editors March 15, 1990.

1980 Mathematics Subject Classification (1985 Revision). Primary 60J55.

Key words and phrases. Brownian motion, local time, Tanaka's formula, modulus of continuity. Research partially supported by National Science Council, ROC, Grant NSC77-0208-M0007-57. 
$g \in C^{2}\left(R^{d-1}\right)$, then we define the local time of $X$ at $C$ to be $L_{t}^{0}(Y)$, where $Y=X^{d}-g\left(X^{1}, X^{2}, \ldots, X^{d-1}\right)$. Let us remark here that the definition is free of ambiguity and able to be extended to more general $(d-$ 1)-dimensional sets by localization. For $g \in C^{2}\left(R^{d-1}\right)$, let $g_{i}=\partial g / \partial x_{i}$, $g_{i i}=\partial^{2} g / \partial x_{i}^{2}$, where $i=1,2, \ldots, d-1 ;$ for $R>0$, let $\|g\|_{R}=$ $\sup \left\{|g(x)|+\sum_{i=1}^{d-1}\left|g_{i}(x)\right|+\sum_{i=1}^{d-1}\left|g_{i i}(x)\right|:|x| \leq R\right\},|\cdot|$ is the usual norm of $R^{d-1}$. Let $B_{s}=\left(B_{s}^{2}, B_{s}^{2}, \ldots, B_{s}^{d}\right), s \geq 0$, be $d$-dimensional Brownian motion. To discuss the continuity properties, we establish first the following moment inequality.

Theorem 2.1. Fix $T, R, m>0$, and let $\tau(R)=\inf \left\{s \geq 0: \sum_{i=1}^{d-1}\left(B_{s}^{i}\right)^{2} \geq R\right\}$. If $f, g \in C^{2}\left(R^{d-1}\right),\|f-g\|_{R}=\delta \leq 1,\|f\|_{R} \leq m,\|g\|_{R} \leq m$, then for any $x \in R^{d}$ we have

$$
E^{x}(L(f, \tau(R) \wedge T)-L(g, \tau(R) \wedge T))^{2 n} \leq C(n, m, T) \delta^{n},
$$

where

$$
L(f, t)=L_{t}^{0}\left(B^{d}-f\left(B^{1}, \ldots, B^{d-1}\right)\right), \quad L(g, t)=L_{t}^{0}\left(B^{d}-g\left(B^{1}, \ldots, B^{d-1}\right)\right)
$$

and $C(n, m, T)$ is a constant depending on $n, m, T$.

Proof. According to the definition, let us write

$$
\begin{aligned}
L(f, \tau(R) \wedge T)= & \left(B_{\tau(R) \wedge T}^{d}-f\left(B_{\tau(R) \wedge T}^{1}, \ldots, B_{\tau(R) \wedge T}^{d-1}\right)\right)^{+} \\
& -\left(B_{0}^{d}-f\left(B_{0}^{1}, \ldots, B_{0}^{d-1}\right)\right)^{+} \\
& -\int_{0}^{\tau(R) \wedge T} I_{\left[B_{s}^{d}>f\left(B_{s}^{1}, \ldots, B_{s}^{d-1}\right)\right]} d B_{s}^{d} \\
& +\int_{0}^{\tau(R) \wedge T} I_{\left[B_{s}^{d}>f\left(B_{s}^{1}, \ldots, B_{s}^{d-1}\right)\right]} d f\left(B_{s}^{1}, \ldots, B_{s}^{d-1}\right) .
\end{aligned}
$$

Applying the Itô formula to the fourth term, we get

$$
\begin{aligned}
L(f, \tau(R) \wedge T)= & \left(B_{\tau(R) \wedge T}^{d}-f\left(B_{\tau(R) \wedge T}^{1}, \ldots, B_{\tau(R) \wedge T}^{d-1}\right)\right)^{+} \\
& -\left(B_{0}^{d}-f\left(B_{0}^{1}, \ldots, B_{0}^{d-1}\right)\right)^{+}-\int_{0}^{\tau(R) \wedge T} I_{\left[B_{s}^{d}>f\left(B_{s}^{1}, \ldots, B_{s}^{d-1}\right)\right]} d B_{s}^{d} \\
& +\sum_{i=1}^{d-1} \int_{0}^{\tau(R) \wedge T} f_{i}\left(B_{s}^{1}, \ldots, B_{s}^{d-1}\right) I_{\left[B_{s}^{d}>f\left(B_{s}^{1}, \ldots, B_{s}^{d-1}\right)\right]} d B_{s}^{i} \\
& +\frac{1}{2} \sum_{i=1}^{d-1} \int_{0}^{\tau(R) \wedge T} f_{i i}\left(B_{s}^{1}, \ldots, B_{s}^{d-1}\right) I_{\left[B_{s}^{d}>f\left(B_{s}^{1}, \ldots, B_{s}^{d-1}\right)\right]} d s .
\end{aligned}
$$

Expressing $L(g, \tau(R) \wedge T)$ in the same way and then taking the absolute dif- 
ference, we get

$$
\begin{aligned}
& |L(f, \tau(R) \wedge T)-L(g, \tau(R) \wedge T)| \\
& \leq\left|f\left(B_{\tau(R) \wedge T, \ldots}^{1}, B_{\tau(R) \wedge T}^{d-1}\right)-g\left(B_{\tau(R) \wedge T, \ldots, B_{\tau(R) \wedge T}^{d-1}}^{1}\right)\right| \\
& +\left|f\left(B_{0}^{1}, \ldots, B_{0}^{d-1}\right)-g\left(B_{0}^{1}, \ldots, B_{0}^{d-1}\right)\right| \\
& +\sum_{i=1}^{d-1} \mid \int_{0}^{\tau(R) \wedge T}\left\{f_{i}\left(B_{s}^{1}, \ldots, B_{s}^{d-1}\right) I_{\left[B_{s}^{d}>f\left(B_{s}^{1}, \ldots, B_{s}^{d-1}\right)\right]}\right. \\
& \left.-g_{i}\left(B_{s}^{1}, \ldots, B_{s}^{d-1}\right) I_{\left[B_{s}^{d}>g\left(B_{s}^{1}, \ldots, B_{s}^{d-1}\right)\right]}\right\} d B_{s}^{i} \\
& +\frac{1}{2} \sum_{i=1}^{d-1} \mid \int_{0}^{\tau(R) \wedge T}\left\{f_{i i}\left(B_{s}^{1}, \ldots, B_{s}^{d-1}\right) I_{\left[B_{s}^{d}>f\left(B_{s}^{1}, \ldots, B_{s}^{d-1}\right)\right]}\right. \\
& \left.-g_{i i}\left(B_{s}^{1}, \ldots, B_{s}^{d-1}\right) I_{\left[B_{s}^{d}>g\left(B_{s}^{1}, \ldots, B_{s}^{d-1}\right)\right]}\right\} d s \mid .
\end{aligned}
$$

Without loss of generality, we may discuss only the case $d=2$ and assume $|x|<R$. Of course,

$$
E^{x}\left(f\left(B_{\tau(R) \wedge T}^{1}\right)-g\left(B_{\tau(R) \wedge T}^{1}\right)\right)^{2 n} \leq \delta^{2 n} \leq \delta^{n}
$$

and

Observe that

$$
E^{x}\left(f\left(B_{0}^{1}\right)-g\left(B_{0}^{1}\right)\right)^{2 n} \leq \delta^{2 n} \leq \delta^{n}
$$

$$
\begin{aligned}
& E^{x} \int_{0}^{\tau(R) \wedge T}\left|I_{\left[B_{s}^{2}>f\left(B_{s}^{1}\right)\right]}-I_{\left[B_{s}^{2}>g\left(B_{s}^{1}\right)\right]}\right| d s \\
& \quad \leq \int_{0}^{T} \int_{-R}^{R} \int_{f(u) \wedge g(u)}^{f(u) V g(u)} \frac{1}{2 \pi s} \exp \left[-\frac{\left(u-x_{1}\right)^{2}+\left(v-x_{2}\right)^{2}}{2 s}\right] d v d u d s, x=\left(x_{1}, x_{2}\right), \\
& \quad \leq \int_{0}^{T}\left(\int_{-R}^{R} \frac{\delta}{\sqrt{2 \pi s}} \exp \left[-\frac{\left(u-x_{1}\right)^{2}}{2 s}\right] d u\right) \frac{1}{\sqrt{2 \pi s}} d s \leq C(T) \delta .
\end{aligned}
$$

So the Markov property will ensure

$$
\begin{aligned}
& E^{x}\left(\int_{0}^{\tau(R) \wedge T}\left|I_{\left[B_{s}^{2}>f\left(B_{s}^{1}\right)\right]}-I_{\left[B_{s}^{2}>g\left(B_{s}^{1}\right)\right]}\right| d s\right)^{n} \\
& \quad \leq E^{x}\left(\int_{0}^{T}\left|I_{\left[B_{s \wedge \tau(R)}^{2}>f\left(B_{s \wedge \tau(R)}^{1}\right)\right]}-I_{\left[B_{s \wedge \tau(R)}^{2}>g\left(B_{s \wedge \tau(R)}^{1}\right)\right]}\right| d s\right)^{n} \\
& \quad \leq n ! E^{x} \int_{0}^{T} \int_{s_{1}}^{T} \cdots \int_{s_{n-1}}^{T} \prod_{i=1}^{n}\left|I_{\left[B_{s_{i} \wedge \tau(R)}^{2}>f\left(B_{s_{i} \wedge \tau(R)}^{1}\right)\right]}-I_{\left[B_{s_{i} \wedge \tau(R)}^{2}>g\left(B_{s_{i} \wedge \tau(R)}^{1}\right)\right]}\right| d s_{i} \\
& \quad \leq n ! E^{x} \int_{0}^{T} E^{\left(B_{s_{1}}^{1}, B_{s_{1}}^{2}\right)} \int_{s_{1}}^{T} \cdots E^{\left(B_{s_{n-1}}^{1}, B_{s_{n-1}}^{2}\right)} \int_{s_{n-1}}^{T}{ }^{n} \times \prod_{i=1}^{n}\left|I_{\left[B_{s_{i} \wedge \tau(R)}^{2}>f\left(B_{s_{i} \wedge \tau(R)}^{1}\right)\right]}-I_{\left[B_{s_{i} \wedge \tau(R)}^{2}>g\left(B_{s_{i} \wedge \tau(R)}^{1}\right)\right]}\right| d s_{i} \\
& \quad \leq C(n, T) \delta^{n} .
\end{aligned}
$$


By the Burkholder inequality, we see

$$
\begin{aligned}
& E^{x}\left\{\int_{0}^{\tau(R) \wedge T}\left|I_{\left[B_{s}^{2}>f\left(B_{s}^{1}\right)\right]}-I_{\left[B_{s}^{2}>g\left(B_{s}^{1}\right)\right]}\right| d B_{s}^{2}\right\}^{2 n} \\
& \quad \leq C(n) E^{x}\left\{\int_{0}^{\tau(R) \wedge T}\left|I_{\left[B_{s}^{2}>f\left(B_{s}^{1}\right)\right]}-I_{\left[B_{s}^{2}>g\left(B_{s}^{1}\right)\right]}\right| d s\right\}^{n} \\
& \quad \leq C(n, T) \delta^{n}
\end{aligned}
$$

and

$$
\begin{aligned}
E^{x}\{ & \int_{0}^{\tau(R) \wedge T}\left(\left(f_{1}\left(B_{s}^{1}\right) I_{\left[B_{s}^{2}>f\left(B_{s}^{1}\right)\right]}-g_{1}\left(B_{s}^{1}\right) I_{\left[B_{s}^{2}>g\left(B_{s}^{1}\right)\right]}\right) d B_{s}^{1}\right\}^{2 n} \\
\leq & C(n) E^{x}\left\{\int_{0}^{\tau(R) \wedge T}\left(\left(f_{1}\left(B_{s}^{1}\right) I_{\left[B_{s}^{2}>f\left(B_{s}^{1}\right)\right]}-g_{1}\left(B_{s}^{1}\right) I_{\left[B_{s}^{2}>g\left(B_{s}^{1}\right)\right]}\right)^{2} d s\right\}^{n}\right. \\
\leq & C(n) E^{x}\left\{\int_{0}^{\tau(R) \wedge T}\left(f_{1}\left(B_{s}^{1}\right)-g_{1}\left(B_{s}^{1}\right)\right)^{2} d s\right\}^{n} \\
& +C(n) E^{x}\left\{\int_{0}^{\tau(R) \wedge T} g_{1}^{2}\left(B_{s}^{1}\right)\left|I_{\left[B_{s}^{2}>f\left(B_{s}^{1}\right)\right]}-I_{\left[B_{s}^{2}>g\left(B_{s}^{1}\right)\right]}\right| d s\right\}^{n} \\
\leq & C(n, T) \delta^{2 n}+C(n, m, T) \delta^{n} \\
\leq & C(n, m, T) \delta^{n} .
\end{aligned}
$$

By the same token, we have

$$
\begin{aligned}
E^{x}\{ & \left.\int_{0}^{\tau(R) \wedge T}\left|f_{11}\left(B_{s}^{1}\right) I_{\left[B_{s}^{2}>f\left(B_{s}^{1}\right)\right]}-g_{11}\left(B_{s}^{1}\right) I_{\left[B_{s}^{2}>g\left(B_{s}^{1}\right)\right]}\right| d s\right\}^{2 n} \\
& \leq C(n) E^{x}\left\{\int_{0}^{\tau(R) \wedge T}\left|f_{11}\left(B_{s}^{1}\right)-g_{11}\left(B_{s}^{1}\right)\right| d s\right\}^{2 n} \\
& +C(n) E^{x}\left\{\int_{0}^{\tau(R) \wedge T}\left|g_{11}\left(B_{s}^{1}\right)\left(I_{\left[B_{s}^{2}>f\left(B_{s}^{1}\right)\right]}-I_{\left[B_{s}^{2}>g\left(B_{s}^{1}\right)\right]}\right)\right| d s\right\}^{2 n} \\
& \leq C(n, T) \delta^{2 n}+C(n, m, T) \delta^{2 n} \\
& \leq C(n, m, T) \delta^{n} .
\end{aligned}
$$

The conclusion follows by lumping the above inequalities together.

Corollary 2.2. Under the assumptions of Theorem 2.1, if $0 \leq s, t \leq T$ then for any $x \in R^{d}$ we have

$$
E^{x}(L(f, \tau(R) \wedge s)-L(g, \tau(R) \wedge t))^{2 n} \leq C(n, m, T)\left(\delta^{n}+|s-t|^{n}\right) .
$$

Proof. Inspecting the proof in Theorem 2.1 , we see

$$
E^{x} \sup _{0 \leq t \leq T}(L(f, \tau(R) \wedge s)-L(g, \tau(R) \wedge t))^{2 n} \leq C(n, m, T)|s-t|^{n} .
$$

Hence

$$
E^{x}(L(f, \tau(R) \wedge s)-L(g, \tau(R) \wedge t))^{2 n} \leq C(n, m, T)\left(\delta^{n}+|s-t|^{n}\right) .
$$

By a well-known theorem of Kolmogorov, Corollary 2.2 implies 
Corollary 2.3. $L(f, t)$ can be modified so that it is jointly continuous in $f$ and $t$ whenever $f$ is restricted in a finite-dimensional subspace of $C^{2}\left(R^{d-1}\right)$.

Let $H$ be the set of hyperplanes in $R^{d}$. Then $H$ can be considered a finitedimensional subset of $C^{2}\left(R^{d-1}\right)$. Therefore we deduce the following result due to Bass [2].

Corollary 2.4. $L(f, t)$ considered as a random field on $H \times[0, \infty)$, can have a continuous version.

\section{Modulus of CONTINUITY}

Since a hyperplane can be expressed as a set of the form $\{x: c x=l\}$, where $x=\left(x_{1}, x_{2}, \ldots, x_{d}\right) \in R^{d}, c=\left(c_{1}, \ldots, c_{d}\right) \in R^{d},|c|=1, l \in R^{1}$, we will write $L(c, l, t)$ instead of $L(f, t)$ whenever dealing with the continuity problem of $L(f, t)$ restricted in $H \times[0, \infty)$. From Corollary 2.4, we know $L(c, l, t)$ is jointly continuous in $c, l$ and $t$. Furthermore, Theorem $2.1 \mathrm{im}-$ plies for every $\alpha, 0<\alpha<\frac{1}{2}$, we have

$$
\left|L(c, l, t)-L\left(c^{\prime}, l^{\prime}, t^{\prime}\right)\right| \leq C\left(\left|c-c^{\prime}\right|+\left|l-l^{\prime}\right|+\left|t-t^{\prime}\right|\right)^{\alpha}
$$

by a more refined version of Kolomogorov's theorem. In the following stronger results will be obtained.

Theorem 3.1. For each $c$ we have almost surely

$$
\varlimsup_{\delta \downarrow 0} \sup _{l} \frac{L(c, l+\delta, t)-L(c, l, t)}{\left(\delta \log \delta^{-1}\right)^{1 / 2}}=2\left[\sup _{l} L(c, l, t)\right]^{1 / 2}
$$

and

$$
\varlimsup_{\substack{0 \leq s \leq t \leq 1 \\ t-s=\delta \downarrow 0}} \sup _{l} \frac{L(c, l, t)-L(c, l, s)}{\left(2 \delta \log \delta^{-1}\right)^{1 / 2}}=1 .
$$

Proof. (3.1) follows from results of McKean [8] and Ray [10]. (3.2) is a result of Csaki, et al. [3].

The problem of getting a modulus of continuity with respect to $c$ is more difficult. We present here a result in several steps. Let $S=\left\{u \in R^{d}:|u|=\right.$ 1\}. Call a finite set $U(\varepsilon)$ and $\varepsilon$-net of $S$, if $U(\varepsilon) \subseteq S,|x-U(\varepsilon)|=$ $\inf \{|x-y|: y \in U(\varepsilon)\} \leq \varepsilon$ for every $x \in S$, and $|x-y| z \varepsilon$ for every $x, y \in$ $U(\varepsilon)$. Obviously, the number of points in $U(\varepsilon)$ is bounded by $C(1 / \varepsilon)^{d-1}$ for a constant $C$ independent of $\varepsilon$ and there exists a sequence $U(1) \subseteq U(2) \subseteq \cdots \subseteq$ $U(n) \subseteq \cdots$ such that $U(n)$ is an $2^{-n}$-net of $S$. Let $I=I_{[0, \infty)}, E=E^{x}$, $P=P^{x}, \quad u B_{s}=\sum_{i=1}^{d} u_{i} B_{s}^{i}, \quad u=\left(u_{1}, \ldots, u_{d}\right) \in R^{d}$.

Lemma 3.2. For each $t \geq 0$ we have almost surely

$$
\varlimsup_{\substack{u, v \in S \\|u-v| \downarrow 0}} \frac{\int_{0}^{t}\left|I\left(u B_{s}\right)-I\left(v B_{s}\right)\right| d s}{|u-v|\left(\log |u-v|^{-1}\right)}<\infty
$$

Proof. From the proof of Theorem 2.1, we see

$$
E\left(\int_{0}^{t \wedge \tau(R)}\left|I\left(u B_{s}\right)-I\left(v B_{s}\right)\right| d s\right)^{m} \leq m ! C(t)^{m}|u-v|^{m}
$$


Hence there exists a constant $c=c(t)>0$ such that

$$
E\left\{\exp \left[\frac{1}{c|u-v|} \int_{0}^{t \wedge \tau(R)}\left|I\left(u B_{s}\right)-I\left(v B_{s}\right)\right| d s\right]\right\}<\infty .
$$

Thus for $b>d-1$

$$
\begin{aligned}
& P\left(\max _{\substack{u \in U(n) \\
v \in U(n+1) \\
|u-v| \leq 2^{-n}}} \int_{0}^{t \wedge \tau(R)}\left|I\left(u B_{s}\right)-I\left(v B_{s}\right)\right| d s \geq b c|u-v|\left(\log |u-v|^{-1}\right)\right) \\
& \quad \leq \sum_{\substack{v \in U(n+1) \\
|u-v| \leq 2^{-n}}} \sum_{u \in U(n)} P\left(\int_{0}^{t \wedge \tau(R)}\left|I\left(u B_{s}\right)-I\left(v B_{s}\right)\right| d s \geq b c|u-v|\left(\log |u-v|^{-1}\right)\right) \\
& \quad \leq C 2^{n(d-1)} e^{-b n} .
\end{aligned}
$$

The Borel-Cantelli lemma tells us

$$
\varlimsup_{n \rightarrow \infty} \operatorname{Max}_{\substack{u \in U(n) \\ v \in U(n+1) \\|u-v| \leq 2^{-n}}} \int_{0}^{t \wedge \tau(R)}\left|I\left(u B_{s}\right)-I\left(v B_{s}\right)\right| d s /|u-v|\left(\log |u-v|^{-1}\right)<\infty, \quad \text { a.s. }
$$

Employing a well-known technique (see Sheu [11] or Knight [7, p. 26]) then letting $R \uparrow \infty$, we can obtain (3.3) from the above inequality.

Lemma 3.3. We have almost surely

$$
\varlimsup_{\substack{u, v \in S \\|u-v| \downarrow}}\left|\int_{0}^{t} I\left(v B_{S}\right) d\left(v B_{S}\right)-\int_{0}^{t} I\left(v B_{S}\right) d\left(v B_{s}\right)\right| /|u-v|^{1 / 2}\left(\log |u-v|^{-1}\right)<\infty .
$$

Proof. Remember that for a continuous square integrable martingale $\left(X_{t}\right)$ with quadratic variational process $\left(\langle X\rangle_{t}\right)$, that is $\left(X_{t}^{2}-\langle X\rangle_{t}\right)$ is a martingale, $\left(\exp \left(X_{t}-\frac{1}{2}\langle X\rangle_{t}\right)\right)$ is a supermartingale. In particular, $E\left[\exp \left(X_{t}-\frac{1}{2}\langle X\rangle_{t}\right)\right] \leq 1$ for all $t$, if $X_{0}=0$. See, for example, Ikeda and Watanabe [5, p.142]. Since $\left.\left(\int_{0}^{t} I\left(u B_{s}\right) d\left(u B_{s}\right)-I\left(v B_{s}\right) d\left(v B_{s}\right)\right]\right)$ is a martingale starting at the origin and its quadratic variational process is $\left(\int_{0}^{t} \sum_{i=1}^{d}\left[u_{i} I\left(u B_{s}\right)-v_{i} I\left(v B_{s}\right)\right]^{2} d s\right)$, we have for any $\alpha, \beta>0, \alpha \beta>d-1$,

$$
\begin{aligned}
P\left(\int _ { 0 } ^ { t } \left[I\left(u B_{s}\right) d\left(u B_{s}\right)-\right.\right. & \left.\left.I\left(v B_{s}\right) d\left(v B_{s}\right)\right] \geq \frac{\alpha}{2} \int_{0}^{t} \sum_{i=1}^{d}\left[u_{i} I\left(u B_{s}\right)-v_{i} I\left(v B_{s}\right)\right]^{2} d s+\beta\right) \\
= & P\left(\alpha \int_{0}^{t}\left[I\left(u B_{s}\right) d\left(u B_{s}\right)-I\left(v B_{s}\right) d\left(v B_{s}\right)\right]\right. \\
& \left.\quad-\frac{\alpha^{2}}{2} \int_{0}^{t} \sum_{i=1}^{d}\left[u_{i} I\left(u B_{s}\right)-v_{i} I\left(v B_{s}\right)\right]^{2} d s \leq \alpha \beta\right) \leq e^{-\alpha \beta} .
\end{aligned}
$$


Since the same bound applies to $-\int_{0}^{t}\left[I\left(u B_{s}\right) d\left(u B_{s}\right)-I\left(v B_{s}\right) d\left(v B_{s}\right)\right]$ as well, we have

$$
\begin{aligned}
& P\left(\left|\int_{0}^{t}\left[I\left(u B_{s}\right) d\left(u B_{s}\right)-I\left(v B_{s}\right) d\left(v B_{s}\right)\right]\right|\right. \\
& \left.\quad \geq \frac{\alpha}{2} \int_{0}^{t} \sum_{i=1}^{d}\left[u_{i} I\left(u B_{s}\right)-v_{i} I\left(v B_{s}\right)\right]^{2} d s+\beta\right) \\
& \quad \leq 2 e^{-\alpha \beta}
\end{aligned}
$$

Now

$$
\int_{0}^{t} \sum_{i=1}^{d}\left[u_{i} I\left(u B_{s}\right)-v_{i} I\left(v B_{s}\right)\right]^{2} d s \leq \int_{0}^{t}\left|I\left(u B_{s}\right)-I\left(v B_{s}\right)\right| d s+t|u-v|^{2} .
$$

Therefore

$$
\begin{aligned}
& P\left(\left|\int_{0}^{t}\left[I\left(u B_{s}\right) d\left(u B_{s}\right)-I\left(v B_{s}\right) d\left(v B_{s}\right)\right]\right|\right. \\
& \left.\quad \geq \frac{\alpha}{2} \int_{0}^{t}\left|I\left(u B_{s}\right)-I\left(v B_{s}\right)\right| d s+\frac{\alpha t}{2}|u-v|^{2}+\beta\right) \\
& \quad \leq 2 e^{-\alpha \beta} .
\end{aligned}
$$

Replacing $\alpha$ and $\beta$ by $\alpha / \sqrt{|u-v|}$ and $\beta \sqrt{|u-v|}\left(\log |u-v|^{-1}\right)$ respectively, we get

$$
\begin{aligned}
& P\left(\frac{\left|\int_{0}^{t}\left[I\left(u B_{s}\right) d\left(u B_{s}\right)-I\left(v B_{s}\right) d\left(v B_{s}\right)\right]\right|}{\sqrt{|u-v|}\left(\log |u-v|^{-1}\right)}\right. \\
& \left.\quad \geq \frac{\alpha}{2} \frac{\int_{0}^{t}\left|I\left(u B_{s}\right)-I\left(v B_{s}\right)\right| d s}{|u-v|\left(\log |u-v|^{-1}\right)}+\frac{t}{2} \frac{|u-v|}{\left(\log |u-v|^{-1}\right)}+\beta\right) \\
& \quad \leq 2|u-v|^{\alpha \beta} .
\end{aligned}
$$

When $n$ is large and $|u-v| \leq 2^{-n}$, we have $\left(\frac{t}{2}\right)\left(|u-v| / \log |u-v|^{-1}\right) \leq 1$. 
Thus

$$
\begin{aligned}
& P\left(\operatorname{Max}_{\substack{u \in U(n) \\
v \in U(n+1) \\
|u-v| \leq 2^{-n}}} \frac{\int_{0}^{t}\left|I\left(u B_{s}\right) d\left(u B_{s}\right)-I\left(v B_{s}\right) d\left(v B_{s}\right)\right|}{\sqrt{|u-v|}\left(\log |u-v|^{-1}\right)}\right. \\
& \left.\geq \operatorname{Max}_{\substack{u \in U(n) \\
v \in U(n+1) \\
|u-v| \leq 2^{-n}}} \frac{\alpha}{2} \frac{\int_{0}^{t}\left|I\left(u B_{s}\right)-I\left(v B_{s}\right)\right| d s}{|u-v|\left(\log |u-v|^{-1}\right)}+\beta+1\right) \\
& \leq P\left(\operatorname { M a x } _ { \substack { u \in U ( n ) \\
v \in U ( n + 1 ) \\
| u - v | \leq 2 ^ { - n } } } \left\{\frac{\int_{0}^{t}\left|I\left(u B_{s}\right) d\left(u B_{s}\right)-I\left(v B_{s}\right) d\left(v B_{s}\right)\right|}{\sqrt{\mid u-v}\left(\log |u-v|^{-1}\right)}\right.\right. \\
& \left.\left.-\frac{\alpha}{2} \frac{\int_{0}^{t}\left|I\left(u B_{s}\right)-I\left(v B_{s}\right)\right| d s}{|u-v|\left(\log |u-v|^{-1}\right)}\right\} \geq \frac{t}{2} \frac{|u-v|}{\log |u-v|^{-1}}+\beta\right) \\
& \leq C 2^{-(\alpha \beta-d+1) n} .
\end{aligned}
$$

By Lemma 3.2 and the Borel-Cantelli lemma, we conclude

$$
\varlimsup_{n \rightarrow \infty} \operatorname{Max}_{\substack{u \in U(n) \\ v \in U(n+1) \\|u-v| \leq 2^{-n}}} \frac{\left|\int_{0}^{t}\left[I\left(u B_{s}\right) d\left(u B_{s}\right)-I\left(v B_{s}\right) d\left(v B_{s}\right)\right]\right|}{\sqrt{|u-v|}\left(\log |u-v|^{-1}\right)}<\infty . \quad \text { a.s. }
$$

Now the proof is completed by the same reason as in Lemma 3.2 .

Theorem 3.4. For each $l, t$, we have almost surely

$$
\varlimsup_{\substack{u, v \in S \\|u-v| \downarrow 0}} \frac{|L(u, l, t)-L(v, l, t)|}{\sqrt{|u-v|}\left(\log |u-v|^{-1}\right)}<\infty .
$$

Proof. Consider the case $l=0$. Then

$$
\begin{aligned}
& |L(u, 0, t)-L(v, 0, t)| \\
& \quad \leq|u-v|\left(\left|B_{t}\right|+\left|B_{0}\right|\right)+\left|\int_{0}^{t}\left[I\left(u B_{s}\right) d\left(u B_{s}\right)-I\left(v B_{s}\right) d\left(v B_{s}\right)\right]\right| .
\end{aligned}
$$

Applying Lemma 3.3, we immediately obtain the result. The proof for the case $l \neq 0$ is identical as for the case $l=0$.

\section{REFERENCES}

1. R. Bass, Occupation times of d-dimensional semimartingales, Sem. Stochastic Process. 1982, Birkhauser, Boston, 1983, pp. 51-76.

2. __ Joint continuity and representations of additive functionals of d-dimensional Brownian motion, Stochastic. Process. Appl. 17 (1984), 211-227. 
3. E. Csaki, M. Csörgö, A Földes, and P. Revesz, How big are the increments of the local time of a Wiener process?, Ann. Probab. 11 (1983), 593-608.

4. D. Freedman, Brownian motion and diffusion, Holden-Day, San Francisco, 1971.

5. N. Ikeda and S. Watanabe, Stochastic differential equations and diffusion processes, NorthHolland, Amsterdam, 1981.

6. K. Itô and H. P. McKean, Diffusion processes and their sample paths, Springer-Verlag, Berlin, 1965.

7. F. Knight, Essential of Brownian motion and diffusion, Math. Surveys, vol. 18, Amer. Math. Soc. Providence, RI. 1981.

8. H. P. McKean, A Hölder condition for Brownian local time, J. Math. Kyoto Univ. Springer, Berlin (1962), 195-201.

9. P. Meyer, Un curs sur les integrals stochastiques, Sem. Probab., Lecture Notes in Math., vol. 511, (1976), 254-394.

10. D. Ray, Sojourn times of a diffusion process, Illinois J. Math. (1963), 615-630.

11. S. Sheu, A simple proof of Chevet's theorem, Bull. Inst. Math. Acad. Sinica, Vol 8, No. 1 (1980), 65-72.

12. M. Yor, Sur la transformee de Hilbert des temps locaux Browniens et une extension de la formule d'Itô, Sem. Probab., Lecture Notes in Math., vol. 920, Springer, Berlin (1981), 238-247.

Institute of Applied Mathematics, National Tsing Hua University, Hsinchu, Taiwan 30043, Peoples's Republic of China 